\title{
XIII.
}

Aus der chirurgischen Universitätsklinik zu Helsingfors.

(Direktor: Prof. Dr. Ali Krogius)

\section{Kasuistische Beiträge zur Pathologie und Therapie des Volvulus des Coecum.}

\author{
Von \\ Dr. R. Faltin, \\ Dozent der Chirurgie.
}

Zoege v. Mante uffel gebübrt das Verdienst, durch seinen Vortrag auf dem Chirurgenkongreß in Berlin 1898 und durch seine späteren Publikationen, namentlich durch seine interessante Monographie vom Jahre 1899, die Aufmerksamkeit der medizinischen Welt auf den Volvulus des Coecum gelenkt zu haben. Eine Anzahl kasuistischer Beiträge wurde in den folgenden Jahren von verschiedenen Autoren publiziert. Ich habe 1902 in der Zeitschrift der Finnländischen Ärztegesellschaft (Finska Läkaresällskapets Handlingar) in schwedischer Sprache eine Studie über diesen Gegenstand veröffentlicht, der eine Zusammenstellung von 75 Fällen zugrunde lag. Unter diesen Fällen befanden sich die 24 von Zoege v. Manteuffel in seiner Monographie angefübrten, weiter 25 aus der mir zugänglichen Literatur gesammelte und schließlich 26 in Finnland (darunter 12 in der Chirurgischen Universitätsklinik zu IIelsingfors) beobachtete Fälle. Später habe ich, unter Zufügung von vier neuen Fällen aus Finnland, eine Umarbeitung in deutscher Sprache meiner obenerwähnten schwedischen Arbeit in Nordiskt Medicinskt Archiv, Abteilung Chirurgie (1902 bis 1903), publiziert. Während des Druckes dieser Arbeit hatte ich die Gelegenheit, zwei neue Fälle zu behandeln. Außerdem wurden mir noch die Krankengeschichten von drei von drei finnischen Kollegen in der letzten Zeit behandelten Fällen zugänglich.

Um die immerhin nicht besonders große Kasuistik zu bereichern, und da diese Fälle Gelegenheit zur Besprechung mancher, nicht nur 
mit den Achsendrehungen des Coecum, sondern auch mit den Darmokklusionen im allgemeinen in Zusammenhang stehenden Fragen darbieten, habe ich beschlossen, die Fälle zu publizieren; meine eigenen etwas ausführlicher, die übrigen in größter Kürze.

\section{Fall 1.}

Okklusion seit $21 / 2$ Tagen. Laparotomie. Detorsion. Coecopexie. Laxantia. Heilung.

Franz Grönkvist, 24jähriger Arbeiter aus Teenala. Aufgenommen am 18. Februar 1903 um 12 Uhr Vorm.

Patient hat sich immer einer guten Gesundheit erfreut. Seit fruhester Kindheit hat er beiderseits Leistenbrttche, die ihm nie Beschwerden gemacht haben. Der rechte Bruch soll 2 Jahre frther als der linke entstanden sein. Vor ca. 7 Jahren erkrankte Patient plötzlich mit heftigen Magenschmerzen, Erbrechen, Obstipation und aufgetriebenem Bauch. Nach einem Tage verschwanden ohne jede Behandlung alle Beschwerden und Patient hat sich völlig gesund gefublt bis zum 16. Febraar $11 \mathrm{Jhr}$ vorm., wo er an einem Anfall, wie der eben beschriebene, wieder erkrankte. An dem erwähnten Morgen war Patient damit beschäftigt die Straße von Eisund Schneemassen zu befreien. Er bediente sich dabei eines $11 / 4 \mathrm{~m}$ langen, im unteren Ende keilförmigen Eisenstabes, den er in der Mitte faßte, vertikal in die Höhe hob, wobei er den ganzen Körper kräftig aufrichtete, und dann mit großer Wucht in die Eismassen stieß, so daß große Blöcke sich abspalteten. Gerade als er mit seinem mit den Eismassen beladenen Schlitten die Straße herunterfuhr, bekam er 80 heftige Bauch. schmerzen, daß er seine Arbeit aufgeben mußte, nach Hause fuhr und sich hinlegte. Die Schmerzen, welche anfallsweise heftiger waren, haben bis jetzt angehalten. Am Morgen hatte Patient einen normalen Stuhlgang, seitdem sind weder Fäzes noch Flatus abgegangen. Am Abend seiner Erkrankung erbrach Patient dreimal, seitdem nicht mehr. Hat aber vor Úbel. keit nichts genießen können.

Status: 19. Februar um 1/29 Uhr abends. Ziemlich kräftig gebauter und gut genährter, junger Mann mit gesunder Gesichtsfarbe. Nicht besonders mitgenommen, aber macht einen geplagten, leidenden Eindruck. Zange belegt. Fötor ex ore. Weder Ubelkeit noch Erbrechen. Lungen, Herz und Nieren zeigen nichts Abnormes. Puls kräftig, regelmäßig, 92 . Temperatur $37^{0}$ resp. $37,4^{0}$ im Rektum.

Bauch etwas aufgetrieben, namentlich in seinem oberen Teile. Wăhrend der Schmerzanfälle, die sich besonders einstellen, wenn der Patient auf dem Rucken liegt oder wenn er sich nach der rechten Seite hin dreht, tritt eine Asymmetrie im Bauch deutlicher hervor. Dieselbe besteht darin, daß die Ileocoecalgegend etwas abgeflacht ist, während von unten nnd rechts eine peristaltisch sich blăhende Walst schräg nach dem linken Hypochondrium hinzieht. Die am meisten prominierende Partie hat ihren Sitz in der linken Halfte des Epigastrium. Das Bild ist etwas verschieden zu verschiedenen Zeitpunkten, aber die Asymmetrie bleibt stets bestehen. Durch Palpation (elastische Resistenz) und Perkussion (dumpfer Schall) 
ist die Asymmetrie ebenfalls ziemlich gat von der Umgebung abgrenzbar. Der Bauch sonst weich. Keine freie Fllissigkeit aufweisbar. Es besteht eine unbedeutende diffuse Druckempfindlichkeit, die in der Nabelgegend und uber der prominierenden Partie etwas stărker ausgeprägt ist. Im linken Hypochondrium kann durch stoßende Palpation ein lautes plätscherndes Geräusch erzeugt werden. Die Bruchpforten frei. Rechts ein mannsfaustgroßer externer, links ein hthnereigroßer interner Leistenbruch.

Patient liegt am liebsten auf der linken Seite mit gegen den Bauch zusammengezogenen Beinen, weil er in dieser Stellung am wenigsten Schmerzen verspurt. Legt er sich auf den Riicken oder dreht er sich im Bette gar um auf die rechte Seite, vermehren sich die Schmerzen dermaßen, daß er laut zu klagen anfängt. Dreht er sich in derselben Richtung weiter, bis er wieder auf der linken Seite zu liegen kommt, werden die Schmerzen erträglicher resp. hören vollständig auf. Patient wird aufgefordert sich einmal nach links herumzudrehen. Die Schmerzen entotehen erst, wenn er von der Bauchlage in die rechte Seitenlage ubergeht, nehmen ab resp. verschwinden wieder, sobald die linke Seitenlage erreicht worden ist. Patient wurde mehrmals aufgefordert, diese Drehongen sowohl nach der einen, wie nach der andern Seite vorzunelımen. Das Resultat war immer das nämliche.

Per rectum können uber 2 Liter Wasser eingegossen werden. Etwas Flatas und einige harte Fäzesklumpen entleeren sich. Patient fuhlt doch keine Erleichterung.

Die Diagnose wird auf Volvulus des Coecum mit Dislokation des Blinddarms nach dem linken Hypochondrium gestellt.

Um 1/410 Uhr Nachm. L a parotomie. Schnitt in der Linea alba, zum gröBten Teile unterhalb des Nabels. Ziemlich viel von einer geruchlosen, sanguinolenten Flitssigkeit entleert sich. Die Hand wird eingefthrt. Die Ileocoecalgegend von Dunndarmschlingen aufgenommen. In der Medianlinie wird eine straffe Mesenterialfalte gefuhlt. Im linken Hypochondrium liegt unterhalb des Zwerchfells und teilweise bis zur linken Lumbalgegend reichend ein sehr großer, ziemlich schlaffer, plätschernder Darm. Mit Schwierigkeit wird derselbe herausgeholt. Es zeigt sich, daß es das Coecum nebst einem Teile des Colon ascendens, ist, welches mitsamt dem untersten ca. $10 \mathrm{~cm}$ vom Ileum $180^{\circ}$ in Linksschraube gedreht ist. Diese Darmteile besitzen ein gemeinsames Mesenterium, welches sich auf dem Colon ascendens bis zur Nähe der Flexura coli hepatia erstreckt und am Coecalrand ein fingerdickes Paket von geschwollenen Lymphdriben besitzt. Der gedrehte Dickdarmteil schlaff, stellenweise, namentlich ca. $20 \mathrm{~cm}$ von der Kuppe des Coecum, ekchymosiert. Detorsion. Wenigstens die Hälfte aller Dănndarmschlingen sind nach rechts ins kleine Becken hinttbergefallen und klemmen durch ihr Mesenterium die Mitte des Colon ascendens ab. Die hinubergefallenen Dunndarmschlingen, die schon recht aufgetrieben sind, werden aus der Bauchhöhle herausgeholt, die Verbältnisse im Bauch nochmals genau gemustert und, nachdem man sich therzeugt hatte, daß kein weiteres Hindernis für die Darmpassage vorlag, nach links vom Colon ascendens in die Bauchhöhle wieder reponiert. Am äußeren Rande des rechten M. rectus wird eine kleine Inzision gemacht und das Coecum daselbst mit einigen 
Catgutnähten (Jodjodkalicatgut nach $\mathrm{Bloch}$ ) an eine Tenia befestigt. Beide Wunden werden exakt durch Etagennähte geschlossen.

Nach der Operation, die ca. 1 Stunde gedauert hatte, war Patient bei gaten Kräften. Kein Erbrechen. Bekam gleich nach dem Erwachen $30 \mathrm{~g}$ englisches Salz in einem Glase Wasser gelöst. Zur Nacht Morphium subkutan.

19. Februar. Ruhig geschlafen. Kein Erbrechen, keine Schmerzen. Flatus abgegangen. Bauch weich, nicht aufgetrieben. Am Morgen wird abermals $30 \mathrm{~g}$ englisches Salz eingegeben. Am Nachmittage entleerten sich ca. $1 \frac{1}{2}$ Liter dunnftussiger, stinkender Fäzes. Der Dickdarm wird mit 4 Liter warmer physiologischer Kochsalzlösung ausgespult.

20. Februar. Fruh am Morgen wird die gleiche Dosis englisches Salz zum dritten Male verabreicht und einige Stunden später hobe Eingießungen mit 4 Liter warmer Kochsalzlösung gemacht. Ziemlich viele dünflussige, stinkende Fäzesmassen entleeren sich im Laufe des Tages.

21. Februar. Am Morgen $25 \mathrm{~g}$ Rizinusöl und einige Standen später Darmsptlung. Abgang von dunnflussigen Stuhlen und Flatus. Bauch weich, vollkommen eingesunken, nicht druckempfindlich.

3. März. Nähte entfernt. Heilung per primam. Durch tägliche Klystiere reichlicher Stuhlgang.

8. Mărz. Temperatur seit 6. März etwas gesteigert. Heute Abend Temperatur $38,2^{\circ}$. Druckempfindlicbkeit im Verlaufe der linken Vena saphena Eisbeutel.

9. März. Das ganze linke Bein geschwollen. Ordination: Elevation,

12. März. Das Bein jetzt sehr geschwollen, ödematős.

14. März. Temperatur wieder normal.

20. März. Das Bein fängt an abzuschwellen. Bleiwasserumschläge. Patient hat offenbar eine schlechte Zirkulation, da seine Haut oft zyanotisch oder marmoriert aussieht.

28. März. Da Patient von seinen Hernien befreit zu werden wtinscht, werden dieselben unter $\mathrm{Schleichscher} \mathrm{Lokalanasthesie} \mathrm{radikal}$ operiert. Rechts ein faustgroßer, externer Leistenbruch, der nach Bassini behandelt wird, der linke interne huhnereigroße Bruch wird ohne Verlagernng des Funikels dadurch verschlossen, daß nach Exstirpation des Sackes die Bauchmuskeln an das Lig. Poupartii vernaht werden. Es erfolgte per primam Heilnng der beiden Wunden.

11. April. Patient darf im Bette sitzen. Das linke Bein kaum mehr geschwollen.

15. April. Darf umhergehen.

Wird am 20. April entlassen. Keine Anschwellung am linken Bein mehr bemerkbar. Patient hat tăgliche normale Stuhle.

\section{Fall 2.}

Okklusion seit 9 Tagen. Laparotomie. Coecum $270^{\circ}$ in Rechtsschraube gedreht und nach dem linken Hypochondrium verlagert. Resektion. Anus praeternaturalis. Laxantia. Heilung.

Ina Sohlberg, 32jährige Arbeitersfrau aus einer Vorstadt Helsingfors. Anfgenommen am 21. Februar vormittags. 
Patientin, welche 3 Kinder hat, das. jungste ist 3 Monate alt, ist nie fruher in ähnlicher Weise wie jetzt erkrankt gewesen. Den 12 . Februar am Nachmittage war Patientin mit Holzhauen beschäftigt. Um ein besonders zähes Stuck Birkenholz spalten zu können, mußte sie sich sehr anstrengen und hieb zuletzt mit Zusammenraffen ihrer ganzen Kraft zweimal auf das Holzstack los. Dabei verspurte sie einen heftigen Schmerz im Bauch und fublte und hörte, als ob etwas unter lautem Kollern sich im Bauch bewegt hätte. Sie versuchte mit der Arbeit fortzufahren, mußte aber bald vor Schmerzen damit aufhören. Die Schmerzen haben seitdem mit wechselnder Intensität angehalten.

Den 13. Februar abends stellten sich UUbelkeiten und Erbrechen ein. Den 14. Februar beobachtete Patientin, daß der Bauch im linken oberen Teile aufgetrieben war. $\mathrm{Da}$ die Schmerzen und das Erbrechen anhielten, besuchte sie einen Arzt, der, ohne die Patientin näher zu untersuchen, Prießnitzsche Umschläge und Opium verordnete. Am 15. und 16. Februar war Patientin gezwungen, die meiste Zeit bettlägerig zu sein. Der linke Teil des Bauches trieb mehr und mehr auf. In der Nacht gegen den 17. Februar erbrach Patientin kolossale Mengen. Bei dem Brechakt sollen Flatus abgegangen sein. Sonst hat seit der Erkrankung totale Verhaltung der Winde nnd der Fäzes bestanden. Am 17. Februar nachmittags wurde ein zweiter Arzt geholt ${ }^{1)}$. Bauch mäßig, links mehr aufgetrieben. Puls und Temperatur normal. Außer der schon angewandten Behandlung verordnete er $\mathbf{z w e i} 1$ Liter große Wasserklystiere, die aber ohne jeden Effekt blieben. Am 19. Februar hatte die Asymmetrie in der linken Bauchhälfte, in der Gestalt einer vertikalen Wulst, solche Dimensionen angenommen, daß die Atmung erschwert war. Der Arzt applizierte selbst ein 1 1/2 Liter großes Klystier. Danach soll etwas Flatus und unbedeutend Fäzes abgegangen, und der Bauch bedeatend kleiner geworden sein. Auch fuhlte sich Patientin fur einige Stunden erleichtert. In den folgenden Tagen wurden zweimal täglich 11/2-2 Liter große Wassereinläufe gemacht. Danach soll immer eine gewisse momentane Erleichterung eingetreten sein. Jedoch versehlechterte sich im ganzen der Zustand der Patientin. Die Kräfte nahmen zusehends ab. Die Bauchschmerzen waren bisweilen sehr heftig, und bemerkte Patientin selbst, daß sie erträglicher in der Rückenlage als in der Seitenlage waren. Der Bauch war wie zuvor asymmetrisch aufgetrieben, ubelriechendes Erbrechen erfolgte mehrmals taglich und Stuhlverstopfung bestand fort. Seit dem 20. Februar konnte Patientin nicht mehr spontan urinieren, sondern mußte katheterisiert werden.

Den 21. Februar vormittags um 12 Uhr wurde Patientin in die chirurgische Klinik eingeliefert, nachdem sie im Schlitten den ca. $5 \mathrm{~km}$ langen Weg nach der Stadt gefahren war.

Status praesens: Gut natriiertes, ziemlich kruftig gebautes Weib. Sieht sehr mitgenommen und leidend aus. Gesicht bleich, bedeckt von kaltem Schweiß, Nase kuhl, Lippen zyanotisch. Kein Halo um die Augen. Zunge etwas belegt, feucht. Kein Foetor ex ore. Puls sehr klein, frequent, 146 in der Minute. Temperatur $38,1^{0}$ resp. $39,4^{0}$ per

1) Die folgenden Angaben stammen teils von ihm, teils von der Patientin. 
rectum. Leukozytose 14000 . Der Harn wird mittelst Katheter entleert, aber leider aus Versehen weggeworfen.

Der Bauch im ganzen bedeutend aufgetrieben, so daß das Epigastrium sich uber dem Niveau des Brustkorbes erhebt. Es besteht eine bedentende Asymmetrie des Bauches, indem die linke Hălfte desselben mehr hervortritt. Besonders erhebt sich gleich links rom Nabel, wo die Asymmetrie ihren höchsten Punkt erreicht, eine nach dem linken Hypochondrium sich hinziehende Wulst. Keine Peristaltik, ist weder sicht- noch hörbar, nicht einmal nach Schttteln oder bei Lageveränderungen der Patientin. Brettharte Muskelspannungen (défence musculaire) nnd große Druckempfindlichkeit uber der Hervorwölbung. Über den thbrigen Regionen besteht ebenfalls eine gewisse Spannung und eine erhebliche Druckempfindlichkeit, so $\mathrm{dah}$ eine genaue palpatorische und perkutorische Untersuchung des Bauches fast unmöglich gemacht wird. Patientin klagt tber fast kontinuierliche, brennende Schmerzen im Racken. Wird Patientin aufgefordert, die Seitenlage, namentlich die rechte Seitenlage, einzunehmen, werden die Schmerzen intensiver und bekommen ihren brennenden Charakter wieder erst, sobald sie sich auf den Rücken gedreht hat. Fur den Augenblick hat Patientin keine thelkeiten oder Erbrechen. Per rectum können nar 1/2 Liter Flẗssigkeit eingegossen werden, ehe Patientin stark zu klagen anfăngt und das Wasser wieder herauslăuft. Uterus klein, retroflektiert, beweglich, die Scheidengewölbe frei, erst bei tieferem Eindrücken empfindlich.

Die Diagnose wurde auf eine Peritonitis, ausgehend von einem Volvulus im Bereiche des Dickdarms, gestellt. Ein Coecum volvulus war am wabrscheinlichsten. Wegen der geringen Quantität der per rectum eingießbaren Wassermenge konnte die Möglichkeit eines Flexurvolvulus nicht von der Hand gewiesen werden.

Laparotomie um 1/2 2 Uhr nachmittags. Vor der Operation wurde 0,2 Koffein und 0,015 Strychnin subkutan injiziert. Äthernarkose. Schnitt in der Linea alba. Därme mit der vorderen Bauchwand leicht verklebt. Nach Lösung der Verklebungen entleert sich ziemlich reichlich sanguinolente, geruchlose Flussigkeit. Die eingefuhrte Hand fuhlt einen kolossal aufgetriebenen Darm, der auf der linken Seite in fast vertikaler Richtung nach dem Hypochondrium sich hinzieht. Ein Teil des Darmes ist nach kräftiger Ekartierung in der Wunde sichtbar, graugrthn, mißfarbig. Vorsichtig wird die gangränöse Schlinge von ihren Verklebungen zu den umgebenden Därmen gelöst und aus der Bauchhöhle herausgewälzt. Es zeigt sich, daß es das enorm aufgeblähte Coecum ist, welches mit einem Teile des Colon ascendens und dem alleruntersten Teile des Ileums eine Drehung in Rechtsschraube von $270^{\circ}$ gemacht hat. Weder auf dem zu-, noch auf dem abfuhrenden Darm kann irgendeine Schntirfurche bemerkt werden. Detorsion. Colon ascendens und transversum blähen sich auf. Der obere Teil des Colon ascendens bildet eine kurze, nach einwärts gelegene Schlinge, die schon ziemlich unbeweglich an der hinteren Bauchwand befestigt ist.

Das untere Colon ascendens besitzt mit dem Coecum und dem Ileum ein gemeinsames Mesenterium, in welchem in der Nähe vom Coecum mehrere geschwollene Lymphdrusen eingelagert sind. Auf der hinteren 
Fläche des Coecum resp. Colon ascendens vereinigen sich drei querlaufende Haustra am Mesenterialansatz za einem bleifederdicken gefäßfuhrenden Strang, der sich am Mesenterium befestigt, nachdem er einen Teil desselben fast zirkulär umschlnngen hat. Durch diese Anordnung wird ein Teil des Mesenteriums zusammengeschnurt und das Coecum resp. Colon ascendens gezwungen, eine Bogenform anzunehmen. Auf der Konvexität des Darmes eine handtellergroße, grülich verfärbte Partie. Auf mehreren Stellen ausgebreitete Serosarupturen.

Mittelst großer Kompressen wird reichlich sanguinolentes Exsadat vom kleinen Becken abgetupft. Nach Absperrung der Bauchhöhle wird vom Dickdarm ein $30 \mathrm{~cm}$ langes und vom Ileum ein ca. $10 \mathrm{~cm}$ langes Stuck reseziert. Die Darmstilmpfe werden durch eine kleine Inzision am Rande des rechten $M$. rectus herausgezogen und daselbst mit Catgutnăhten parallel befestigt. Der Colonstumpf wird mit einem Seidenfaden umgebunden, in den Ileumstumpf wird ein dickes Drainrohr eingeschoben und sorgfaltig so befestigt, daß kein Darminhalt am Rohr vorbeifließen konnte.

Die Bauchhöhle wird nochmals durchgemustert, ohne daB irgendein weiteres Hindernis hătte gefunden werden können. Die Dunndărme sind teilweise zusammengefallen, teilweise mäßig geblältt. Während der ganzen Operation prolabierten keine Dünndarmschlingen. Das Mesenterium war lang und schlaff. Die Radix desselben schien sich bis zur Nähe des Promontoriums zu erstrecken. Năher wurden die Mesenterialverhältnisse nicht aufgeklärt, da es galt, so schnell wie möglich die Operation abzuschließen.

Die mediane Bauchwunde wurde bis anf den untersten Winkel, wodurch ein $5 \mathrm{~cm}$ breiter Silbergazestreifen (Crédé) nach dem kleinen Becken gefthrt wurde, in Etagen vernäht.

Nach der Operation, die ca. 1 Stunde gedauert hatte, erwachte die Patientin sehr bald. Der Puls war frequent, aber nicht schlechter als vor der Operation. Da kein Erbrechen, wurde $30 \mathrm{~g}$ englisches Salz, in $100 \mathrm{~g}$ Wasser gelöst, verabreicht. Etwa 2 Stunden später verschlechterte sich der Zustand, wesbalb $1000 \mathrm{ccm}$ Kochsalzlösung intravenös gegeben wurden. Danach wurde der Puls kräftig. Zur Nacht 0,01 g Morphium und Kampfer und Koffein subkutan jede dritte Stunde.

Musterung des resezierten Darmes. Der große, $32 \mathrm{~cm}$ lange und $18 \mathrm{~cm}$ breite, bogenförmig gekrtimmte Sack enthält außer Gasen ca. 1 Liter ubbelriechender dunnflussiger Fäzes. Längs der ganzen Konvexität ausgedehnte Ulzerationen in der Schleimhant, auf mehreren Stellen gehen die Geschwtire fast bis anf die Serosa.

Untersuchung der Aszitesflussigkeit. Beim Sedimentieren setzt sich ein roter Bodensatz ab, der aus roten Blutkörperchen und einzelnen Endothelzellen und polynakleären Lenkozyten besteht. Bakterien können trotz genauen Suchens nicht gefunden werden. Mittelst sterilisierter Pjpette werden ca. $2 \mathrm{ccm}$ von der Aszitesflussigkeit in drei anaerobe Zuckeragarröhrchen und ein aerobes Bouillonröhrchen verteilt. Das letzte bleibt steril, aber in den anaeroben Kulturen gehen 1-10 kleine, wetzsteinförmige Kolonien auf, die aus ziemlich großen, ovalen Diplokokken bestehen. Die Kokken bilden mitunter kurze Ketten, 
wachsen auch aerob, auf Glyzerinagar in kleinen punktförmigen Kolonien, trtben die Bouillon, koagulieren die Milch, aber wachsen nicht bei Zimmertemperatur auf Gelatine. Näher wurden die Kokken nicht verfolgt.

22. Februar. Hat rubig die ganze Nacht durchgeschlafen. Reich. lich Flatus und Fazes (ca. 2 Liter) haben sich entleert. Der Bauch weich, nicht empfindlich, bedeutend kleiner. Am Morgen wird $30 \mathrm{~g}$ englisches Salz und etwas später $0,01 \mathrm{~g}$ Strychnin (subkutan) versbreicht. Patientin genießt etwas Bouillon, aber erbricht es bald wieder. Am Nachmittage wird der umgebundene Colonstumpf punktiert und durch die Nadel ca. $500 \mathrm{ccm}$ warmer physiologischer Kochsalzlösung injiziert. Im Verlaufe der Nacht sollen drei warme Einlaufe per rectum zu je $300 \mathrm{ccm} 1 \mathrm{E} ß$ löffel Digitalisinfusion gegeben werden. Außerdem Mor. phium. Im ganzen hat sich durch den Schlanch seit der Operation 3 Liter Fäzes entleert.

23. Februar. Der Bauch eingesunken. Pals krăftig. Guter allgemeiner Zustand. Am Morgen wird $30 \mathrm{~g}$ englisches Salz verabreicht und während des Tages $3 \times 300 \mathrm{ccm}$ physiologischer Kochsalzlösung per rectum und ca. 1 Liter Do. durch den Colonstumpf eingegossen. Durch den Schlauch haben 1,5 Liter Fazes sich entleert.

24. Februar. Zustand wie gestern. Englisches Salz und Kochsalzlösung per rectum und durch den Colonstumpf wie gestern. Zirka 2 Liter Fäzes sind abgegangen.

25. Februar. Der Silbergazestreifen wird entfernt und ein kleiner Jodoformgazetampon nur durch die Bauchdecken eingefthrt.

26. Februar. Befinden sehr gut.

27. Februar. Die Dupuytrensche Darmklemme und eine Kochersche Arterienzange werden um den Sporn angelegt, so daß der ganze außerhalb des Bauches belegene Teil desselben abgeklemmt wird.

3. März. Täglich sind ca. 1 Liter Kochsalzlösung, teils per rectum, teils durch den Colonstumpf verabreicht worden. Seit heute wird davon bis aufs weitere Abstand genommen, da Patientin jetzt genligend Fldssigkeit per os zu sich nimmt. Die Darmklemme und die Arterienzange haben den Sporn durchschnitten, sie werden jetzt tiefer angelegt. Etwas Flatus ist per rectum abgegangen. Die Nahte werden entfernt. Heilung per primam.

4. März. Die Darmklemme hat sich gelöst, wird wieder etwas tiefer angelegt.

7. März. Im untersten Teil der medialen Wunde hat sich ein Abszeß gebildet, der geklafft und austamponiert wird. Die Darmklemme wird zum vierten Male angelegt.

9. März. Die Darmschere wird zum fünften Male angelegt, noch immer auf den außerhalb der Bauchhöhle belegenen Teil des Sporns, der allmählich in seiner ganzen Breite durchgequetscht worden ist.

14. März. Mikulicz' Kentrotrib wird tief auf den intraperitoneal belegenen Teil des Sporns appliziert.

15. März. Patientin hat sehr taber Schmerzen und Spannnng im Leibe geklagt. Nach einigen Tagen hatte das Instrument den tiefsten Teil des Sporns durchgequetscht. Eine kleine, nach außen belegene Brucke wird mit dem Kentrotrib gefaßt. Nach einigen Tagen fiel das Instrument heraus. 
Am 26. März erfolgte ein Stull per vias naturales. Den 28. März wurde ein Versuch gemacht, den Anus praeternaturalis obne die Eröffinung der Bauchhöhle zu schließen. Mit zwei Reihen fortlaufender Seide wurden die Darmstumpfe verschlossen. Nach der Naht ragte der genăbte Darm von der Bauchwand heraus, als ein $4 \mathrm{~cm}$ breiter und $3 \mathrm{~cm}$ langer Körper.

3. April. Die Nähte hielten nicht, sondern schnitten durch und der Anus praeternaturalis bekam sein altes Aussehen wieder. Doch erfolgte Stuhlgang nur per vias naturales.

Am 16. April wird der Kentrotrib nochmals angelegt, wonach wiederum fur einen Tag Schmerzen and Ubelkeiten entstanden. Am 22. April war der Kentrotrib herausgefallen.

24. April. Suturatio anus praeternaturalis. In Narkose wird der After umschnitten und mobilisiert. Die Bauchböhle wird dabei auf einigen Stellen eröffnet, aber durch Tampons abgesperrt. Die prolabierende Darmsehleimbaut wird entfernt. Danach Invagination und Vernăhung des Darms mit drei Reihen fortlaufender Seide.

Der vernähte Darm wird in die Bauchhöble versenkt und die Bauchwunde bis auf eine kleine Stelle, wodurch ein Gazestreifen auf die Naht eingefulhrt wird, geschlossen.

10. Mai. Reaktionsloser Verlauf. Patientin darf im Bette sitzen.

13. Mai. Patientin darf mit Bauchbinde umbergehen. Der Darm funktioniert tadellos.

22. Mai. Wird geheilt entlassen. Hat an Gewicht zugenommen. Keine Störungen seitens des Darmes. Eine kleine granulierende Stelle besteht noch am untersten Ende der Narbe.

Hier lasse ich die drei von meinen finnischen Kollegen behandelten Fälle folgen.

Dr. John Sonck aus Wiitasaari teilt mir brieflich folgenden Fall mit, dessen Krankengeschichte in verkürzter Form hier wiedergegeben wird.

\section{Fall 3.}

Coecum im linken Hypochondrium ca. $360^{\circ}$ in Rechtsschraubegedreht. Tod nach ca. 21/2 Tagen ohne Operation.

Matti Waris, 16 jähriger Banernsohn aus Wiitasaari. Eingeliefert im Krankenhaus des Kirchspiels am 24. September.

Früher stets gesund gewesen, namentlich keine Störungen seitens des Magendarmkanals. Am 22. September war Patient bei der Heuernte beschäftigt. Er stand dabei oben auf dem Heuschober vorntiber und abwärts gebeugt, mit dem Bauch an ein querverlaufendes Holz gelehnt. Spurte die folgende Nacht gelinde Banchschmerzen. Ging trotz des Unwohlseins die folgenden Tage in die Arbeit. Nach dem Frubstttck, als er sich zur Ruhe hingelegt hatte, wurde er plötzlich von heftigen Bauchschmerzen und Stuhldrang taberfallen. Es erfolgte eine normaler Stuhl. Bald stellte sich Erbrecben ein. Prießnitzsche Umschläge und $\mathrm{O}_{\mathrm{pi}_{\mathrm{m}}}$ 
blieben ohne Erfolg. Am 24. September wurde der Patient nach dem Krankenhause gebracht.

St a tu s: Sehr kräftiger, muskulöser Jungling. Mitgenommen. Leidender Gesichtsausdruck. Puls 120. Temperatur normal. Bauch mäßig aufgetrieben, asymmetrisch, indem ein lokaler Meteorismus von der rechten Fossa iliaca schräg nach dem linken Hypochondrium sich hinzieht. Keine Peristaltik sichtbar. Unbedeutende Druckempfindlichkeit. Keine freie Flussigkeit. Fäkales Erbrechen. Absolute Obstipation. 1/2 Liter Flüssig. keit kann per rectum eingegossen werden.

Eine vorgeschlagene $O$ peration wurde bestimmt vom Patienten abgelehnt. Deswegen muste die Behandlung auf hohe Wassereinläufe und Morphium eingeschränkt werden. Am 25. September verschlechterte sich der Zustand sichtbar. Puls 130-140. Kopiöses, fäkulentes Erbrechen. Die asymmetrische Auftreibung !im Leibe wurde noch stärker hervortretend. Nunmehr konnte nur $1 / 2-1$ Liter unter bedeutenden Schmerzen eingegossen werden. Am Nachmittage willigte Patient schließlich in eine Operation ein. Bei den Vorbereitungen zur Operation verschied aber Patient. Die Sektion zeigte folgendes. Reichlich blutiger Aszites. In der Mitte des Bauches liegt ein schrigg vom linken Hypochondrium, von unterhalb des Rippenbogens nach der rechten Fossa illaca hinziehender, kolossal aufgetriebener, dunkelblanroter Dickdarm, der erst nach Punktion herausgewälzt werden kann, trotzdem der Schnitt vom Proc. xiphoid. bis zur Symphyse reichte. Die aufgetriebene Darmschlinge bestand aus dem untersten Teile des Ileum, aus dem Coecum und einem Teil des Colon ascendens, welche ca. $360^{\circ}$ in Rechtsschraube gedreht waren. Auf mehreren Stellen Serosarupturen. Die tlbrigen Därme waren nicht aufgetrieben.

Epikrise: Dieser Fall zeigte ein für Coecum volvulus so charakteristisches Bild, daß ein per Telephon konsultierter Chirurn nur auf Grund der Beschreibung die richtige Diagnose stellte und zu sofortiger Operation riet. Leider konnte der gute Rat nicht befolgt werden, da der Patient und seine Angehörigen anfangs absolut gegen jede Operation waren.

Dr. A. Sundholm aus Forssa hat die Liebenswürdigkeit gehabt, mir folgenden interessanten und gut beobachteten Fall ausführlich brieflich mitzuteilen. In etwas verkürzter Form gebe ich hier die Krankengeschichte nebst einigen Reflexionen des Mitteilers wieder.

Fall 4.

Okklusion seit ca. 10 Stunden. Laparotomie. Coecum am Beckeneingange, $11 / 2 \mathrm{mal}$ in Rechtsschraube gedreht. Typhlostomie. Drainage der Bauchböhle. Tod nach 6 Tagen. Coecum gangränös.

Joseph Kuusniemi, 25jähriger Bauer aus Jockis. Soll während der letzten Jahre mehrmalg an plötzlich auftretenden Bauchschmerzen namentlich rechts vom Nabel gelitten haben. 
Erkrankte am 18. Oktober 1902 um 2 Uhr in der Nacht mit heftigen Bauchschmerzen, Erbrechen und Diarrhöe. In der Nacht erfolgten drei wässrige Stthle. Patient kam um 9 Uhr morgens in die Sprechstunde des Herrn Dr. A. Sundholm.

Status praesens. Kräftig gebauter Mann. Sehr angegriffen, verfallenes, mit kaltem Sehweiß bedecktes Gesicht, Ausdruck angstlich. Puls fadenförmig. Zunge feucht. Leberdämpfung verschwunden. Bauch klein, bretthart gespannt, besonders rechts vom Nabel, therall etwas druckempfindlich. Der Patient wurde gleich in das von Dr. S u n $\mathrm{d} \mathrm{holm}$ geleitete Krankenhans tberfuhrt. Als der Patient am 1/210 Uhr vormittags nochmals untersucht wurde, war der Bauch etwas aufgetrieben, Darmschlingen waren in der Mitte und auf der linken Seite sichtbar. Keine freie Flitssigkeit anfweisbar. Uberall tympanitischer Perkussionston. Puls sehr klein, 68. Temperatur $36,1^{0}$ resp. $37,9^{0}$ im Rektum. Um 11 Uhr vormittagg war der Bauch sehr stark aufgetrieben, einzelne Schlingen zeichneten sich ab in der Mitte des Bauches. Intensive Bauchsehmerzen.

„Die Diagnose wurde anfangs anf ein perforiertes Ulcus ventriculi seu duodeni oder auf eine perforative Appendicitis gestellt. Da eine genaue Anamnese besonders in bezug auf die fruberen Anfalle infolge des angegriffenen $Z$ ustandes des Patienten anfangs nicht erhalten werden konnte und weil bei dem Patienten noch nach dem schweren plötzlichen Erkranken in der Nacht drei wässrige Stühle erfolgt waren, schien mir eine Darmokklusion vollkommen ausgeschlossen, besonders da die Bauchdecken bretthart gespannt waren. Als später die Spannung etwas nachließ nnd Darmschlingen sichtbar wurden, fing ich an meine erste Diagnose zu bezweifeln. Jedenfalls schien mir ein ungesäumter operativer Eingriff dringend geboten" 1).

Laparotomie um $12 \mathrm{Jhr} 15$ Minuten. Mediansehnitt oberhalb des Nabels. Man stöbt gleich auf einen dunkelblauen, ballonförmigen, kolossal aufgetriebenen Darm, der erst nach Erweiterung der Bauchwunde abwärts vorsichtig herausgewälzt werden kanu. Gleichzeitig entleert sich reichlich ubelriechende, sanguinolente Aszitesflussigkeit. Der ballonförmig geblähte Darm ist das $1 \frac{1}{2} \mathrm{mal}\left(=360^{\circ}+180^{\circ}\right)$ in Rechtssehraube gedrehte Coecum nebst Colon ascendens. Das Coecum liegt am Beckeneingang in der Mitte, das Colon ascendens verlänft nach oben außen. An der Flexura coli hepatica eine deutlich ausgeprägte Schnurfurche. Das Mesocoecum ist ebenso wie der Dickdarm blansehwarz bis hinauf zum Colon transversum. Die Dunndärme leer und zusammengefallen. Detorsion. Dabei entstehen einige Serosarupturen am Coecum. Da es unmöglich war, den gedreht gewesenen Dickdarm zu reponieren, und der Patient fast pulslos war, so daß eine Resektion ausgeschlossen erschien, wurde am Coecum eine Enterostomie nach Witzel auggefuhrt. Reichlich Gase und blutiger, dünnflussiger Darminhalt entleeren sich sogleich. Das jetzt kollabierte Coecum wird reponiert und die Fistelöffnung in die bis auf eine kleine Stelle vernähte Bauch wunde befestigt. Am Rande des rechten Musculus rectus wird eine Kontrainzision verlegt und ein großer Miku li c z scher Tampon gegen das kleine Becken eingefuhrt.

1) Wörtliche Übersetzung aus der brieflichen Mitteilung von Dr. Su ndholm. 
Nach der Operation werden teils Wassereinläufe, teils große Darmspulungen vorgenommen. Um $6 \mathrm{Ohr}$ wird eine $1600 \mathrm{ccm}$ große intravenöse Kochsalzinfusion gegeben. Jede Stunde Kampfer subkutan.

19. Oktober. Schmerzen im Bauch, der mehr aufgetrieben ist. Durch äie Fistel sind reichlich Gase und sanguinolenter Darminhalt abgegangen. Der Verband wird mehrmals am Tage gewechselt wegen der starken, tbelriechenden Sekretion durch die tamponierte rechte Wunde. Intravenöse Kochsalzinfusion. Puls nicht fuhlbar.

20. Oktober. Der Puls wieder fühlbar. Die Fistel funktioniert tadellos.

21.-22. Oktober. Der Zustand unverändert. Kein Erbrechen, aber Aufstoßen. Der Magen erscheint gebläht.

24. Oktober. Äußerst elend. Der Magen sehr stark hervortretend. Aus der rechten Wunde fließt fäkulente Flussigkeit. Der durch die Wunde sichtbare Darm ist gangränös. Die Bauchschmerzen sehr heftig. Kein Erbrechen. Abends um 7 Uhr 30 Min. starb der Patient.

Die Sektion zeigt eine Gangrän des Coecum und Colon ascendens. Diese Darmteile haben ein ca. $1 / 2 \mathrm{~cm}$ dickes, ca. $8 \mathrm{~cm}$ langes, oben etwas kutreres Mesenterium mit thrombosierten Gefäßen. Die Flexura coli hepatica ist an der Leber und der Gallenblase aufgehängt. Hierdurch wird das Mesocolon so straff gespannt, daß das Duodenum wie durch einen Strang komprimiert wird. Der Ventrikel sehr stark gebläht und ausgedehnt, die große Kurvatur reicht bis zum Nabel. Die Jejunumschlingen etwas gebläht, injiziert und zusammengeklebt, die Ileumschlingen meistens zusammengefallen.

Epikrise. Dieser Fall ist in mehrerer Beziehung sehr interessant. Schon das heftige Einsetzen der Symptome, wodurch eine Perforationsperitonitis vorgetäuscht wurde, ist beim Coecumvolvulus sehr ungewöhnlich. Ich werde hierauf später noch zurückkommen. Bemerkenswert ist auch die während der drei Stunden vor der Operation beobachtete rapide Zunahme des Meteorismus. Es ist selten dem Chirurgen die Gelegenheit geboten, die Entwicklung dieses Symptomes so im Beginne der Krankheit zu verfolgen. Als ein recht seltenes Vorkommnis sei noch die Kompression des Duodenum erwähnt, die hier durch das infolge Schwellung und Verwachsungen der Flexura coli hepatica straff gespannte Mesocolon ascendens zustande gekommen war. Zoege v. Manteuffel hat in seiner Kasuistik zwei Fälle (Nr. 1 und Nr. 14), wo das Duodenum durch das Coecum komprimiert wurde, in dem einen Falle war das Duodenum noch dazu gedreht.

Hier lasse ich noch ein kurzes Referat eines von Dr. Sahlberg in der finnischen medizinischen Zeitschrift Duodecim, 1902, Nr. 10 in finnischer Sprache publizierten Falles folgen. 
Fall 5.

Okklusion seit 11 Tagen. Laparotomie. Coecum in der linken Fossa iliaca. Darmpunktion. Laxantia. Heilung.

K. K., 35 jähriges Bauernweib aus Kemi. Aufgenommen ins Krankenhaus in Torneå am 1. September 1902. Während der letzten Jahre an Obstipation gelitten, so daß sie gezwungen gewesen ist, Abführmittel zu gebrauchen. Seit einigen Monaten hat der Darm normal funktioniert. Auch sonst gesund gewesen. Hat 5 Kinder geboren. Erkrankte ohne nachweisbare Ursache plötzlich am Vormittage den 22. August mit heftigen brengenden Schmerzen in der rechten unteren Bauchhälfte. Der Bauch trieb auf. Klystiere ohne Erfolg. Erbrechen einige Male am Nachmittage, aber seitdem kein Erbrechen bis zum 28. August, wo es sich wieder ein. stellte und übelriechend wurde. Der Bauch trieb mehr und mehr auf. Die Schmerzen nahmen zu. Tägliche Klystiere blieben erfolglos.

Statu s bei der Aufnahme am 1. September. Gewöhnlicher Körperbau. Angegriffen. Temperatur normal, Puls 70. Zunge trocken, belegt. Banch aufgetrieben, Peristaltik sichtbar. Überall etwas Druckempfindlichkeit, besonders in der Nabelgegend und auf der linken Seite.

Sogleich wurde der Magen ausgespült, gelbliche, schleimige Flüssigkeit. Einige, ca. 3 Liter große Wassereinläufe bringen Gase und etwas Fäzes mit sich. Patientin fühlt sich erleichtert. Bauch etwas kleiner. Am Abend wieder Verschlechterung. Klysmata ohne Erfolg. Am 2. September ist der Bauch wieder mehr aufgetrieben. Da nochmals applizierte große Klystiere erfolglos blieben, wurde um $10 \mathrm{Uhr}$ des Morgens zur Laparotomie geschritten. Medianschnitt. Etwas sanguinolente Aszitesflüssigkeit. Sämtliche Dündarmschlingen sehr gebläht. Quer thber den Bauch verläuft nach der linken Fossa iliaca das $180^{\circ}$ von rechts nach links gedrehte, blaurote, stark geblähte Coecum nebst Colon ascendens. Mit Schwierigkeit wird der Darm heransgeholt. Weder durch Druck auf den Sack noch durch einen per rectum hoch hinauf eingeschobenen Schlauch läßt der Darm sich entleeren. Deswegen Panktion des Coecum. Reichlich stinkender flussiger Inhalt und Gase strömen heraus. Auch von den untersten Ileumschlingen wird der Inhalt vorsichtig herausgedrïckt. Sutur der Punktionsöffnung. Desinfektion mit Sublimatlösung. Trockene Bauchtoilette. Reposition der Därme. Bauchnaht.

Gleich nach der ca. $3 / 4$ Stunde dauernden Operation wird ca. $700 \mathrm{ccm}$ physiologische Kochsalzlösung intravenös gegeben. Um 2 Uhr ein kleines Klystier, wodurch Abgang von Gasen und etwas Fäzes. Um 6 Uhr wird $15 \mathrm{~g}$ Magnes. sulphur. in $1 / 2$ Glas Wasser verabreicht.

3. September. Hat eine Stuhlentleerung gehabt. Banch jetzt eingesunken. Der weitere Verlauf ungestört. Wunde p. p. geheilt. Wird am 23. September geheilt entlassen.

Epikrise. Es handelte sich in diesem Falle unzweifelhaft um eine Obturation, die, wie auch Sahlberg in der Epikrise vermutet, wahrscheinlich nicht einmal zu einer vollständigen Unwegsamkeit des Darmlumens geführt hatte, da ja nach den ersten, im Kranken- 
hause gegebenen Klysmen noch Abgang von Winden und Kot erfolgte. Der Operationsbefund ergab auch, soweit nach der Beschreibung beurteilt werden kann, daß eine Verlagerung oder Umknickung nach innen mit Drehung von nur ca. $180^{\circ}$ um die Darmachse bestand. Die Nutrition der Darmwand hatte offenbar dadurch gar nicht gelitten.

Bemerkenswert ist, daß dieser Fall, trotzdem er erst am 11. Tage nach Einsetzen der Symptome zur Operation kam, dennoch in Genesung überging. Soweit mir bekannt, sind nur wenige Fälle von Coecumvolvulus nach ebenso langer oder längerer Krankheitsdauer geheilt worden. Dies sind die Fälle von Weinreich, operiert am 11. Tage, Romm, Philipowicz, beide operiert am 16. Tage und ein Fall von Obalinski (Geitel Feuer) mit nicht genau zu bestimmendem Beginn. Ich zögere nicht, den glücklichen Ausgang in dem Falle 5, neben den obenerwähnten günstigen Umständen, der ausgiebigen Entleerung des Darmes sowohl bei, wie nach der Operation zuzuschreiben.

Im ganzen ist es mir also gelungen, Krankengeschichten über 35 Fälle von Volvulus des Coecum aus Finnland zu sammeln. Kommen noch dazu 3 Fälle, die mir nur nach mündlicher Mitteilung bekannt sind und über die ich aus verschiedenen Gründen keine näheren Angaben erteilen kann. Beobachtet sind also in Finnland im Verlaufe von einigen Jahren ${ }^{1)}$ wenigstens 38 Fälle von der erwähnten Krankheit.

Wie ich in meiner schon erwähnten Abhandlung hervorgehoben habe, ist es, wenigstens nach Literaturstudien zu urteilen, sehr wahrscheinlich, daß verschiedene Rassen eine verschiedene Disposition zu Achsendrehungen des Darms und speziell zu Volvulus des Coecum besitzen und weiter, daß die finnische Nation in dieser Beziehung eine der ersten Stellen einnimmt. Es ist nicht ganz leicht, eine befriedigende Erklärung dieses auffallenden Verhältnisses zu geben. Eine Täuschung insofern, daß die hohe Volvulusfrequenz in Finnland nur eine scheinbare wäre, scheint mir ausgeschlossen. Es ist nämlich kaum denkbar, daß z. B. aus Deutschland, wo doch das jeder aktuellen Frage zugewandte Interesse besonders rege zu sein pflegt, seit den Publikationen von Z. v. Mante uffel nicht größere Kasuistiken veröffentlicht worden wären, wenn nur ein diesbezügliches Material zur Verfügung gestanden hätte. Dann bleibt einem eben nichts anderes übrig, als betreffend die finnische Nation eine wirkliche erhöhte Dis-

1) Der älteste Fall stammt aus dem Jahre 1888. Bis 1898 sind nur 5 Fälle bekannt geworden. Die übrigen 33 Fälle stammen also aus den letzten 6 Jahren. 
position für Achsendrehungen im allgemeinen und für Coecumvolvulus im speziellen anzunehmen.

Diese Disposition kann wahrscheinlich wie jede andere teils kongenitaler, teils erworbener Natur sein. Was die erstere betrifft, besteht sie in der durch 'das Vorhandensein eines Mesenterium ileocoeci com. bedingte abnorme Beweglichkeit des Coecum. Ob die Finnländer wirklich ein bewegliches Coecum öfter besitzen als andere Völker, ist zwar noch nicht festgestellt. Es wäre deswegen eine interessante Aufgabe, durch vergleichende Untersuchungen an einem größeren Leichenmaterial die Beweglichkeitsverhältnisse des Blinddarms sowohl bei Finnländern wie bei anderen Rassen festzustellen. Würde es sich wirklich herausstellen, daß die Finnländer relativ oft ein Mesenterium ileo-coeci com. besitzen, hätte man mit einer Rasseneigentümlichkeit zu tun, die wahrhaftig nicht schmeichelhaft für den Besitzer wäre, denn dieselbe deutet unzweifelhaft auf eine niedrige Entwicklungsstufe hin.

Bemerkenswert ist, daß die Mehrzahl (ca. 70 Proz.) der von Coecumvolvulus betroffenen Patienten sich in einem verhältnismäßig jungen Alter, diesseits der 40er Jahren befinden, während beim Flexurvolvulus nach Treves und Edgren umgekehrt die meisten Patienten mehr wie 40 Jahre alt sind. Nicht zu vergessen ist noch, daß ganz wie beim Coecumvolvulus (F a lt in) ebenfalls beim Flexurvolvulus (Treves, Edgren, Kuhn) in den meisten Fällen über frühere verschiedenartige Darmstörungen resp. über mehr oder weniger heftige Ileuserscheinungen berichtet wird. Alle diese Lmstände scheinen mir ebenfalls dafür zu sprechen, daß beim Coecumvolvulus kongenitalen Einflüssen, die schon in jüngeren Jahren zum Vorschein kommen können, eine größere ätiologische Bedeutung zukommt als beim Flexurvolvulus, wo das meistens hohe Alter der Patienten auf allmählich erworbene Zustände hindeutet. Allerdings sollen nach Budberg und Koch auch beim Flexurvolvulus kongenitale Verbältnisse in erster Linie die prädisponierenden Momente abgeben.

Was die erworbene Disposition anbelangt, glaube ich nicht, daß ihre Bedeutung hoch anzuschlagen ist. In Finnland gehören Ventrikelaffektionen zu den allergewöhnlichsten Krankheiten, bilden gewissermaßen die nationelle Krankheit. Die unzweckmäßige, schlecht zubereitete Nahrung (saures Brot, saure Milch, Kartoffeln, stark gesalzene rohe Fische usw.) der Bevölkerung erseheint auch besonders geeignet Ventrikel- und Darmstörungen hervorzurufen. Vielleicht können diese Umstände dazu beitragen, eine schon vorhandene kongenitale Disposition zu erhöhen. In welcher Weise dies in bezug auf das Coecum geschieht, mag dahingestellt sein. 
In meiner erwähnten Arbeit habe ich darauf aufmerksam gemacht, daß Männer viel öfter als Weiber erkranken, eine Eigentümlichkeit, die in bezug auf andere Arten von Achsendrehungen, wie z. B. für den Flexurvolvulus längst bekannt ist. In meiner letzten Zusammenstellung waren c. 70 Proz. Männer und 30 Proz. Weiber. Besonders hervorzuheben ist, daß ein großer Prozentsatz (ca. 40 Proz.) sämtlicher Patienten aus jungen Männern bestand, die im Alter von 17-30 Jahren waren und daß dieser Umstand bei der Differentialdiagnose zwischen Volvulus der Flexur und des Coecum verwertet,werden kann. Auch in den eben mitgeteilten 5 Fällen kommen diese Verhältnisse zum Vorschein, indem $3 / 5$ der Patienten junge Männer waren.

Während die Männer gewöhnlich im 3. oder am Ende des 2. Dezenniums an Coecumvolvulus erkranken, erreicht das weibliche Geschlecht ihr Frequenzmaximum in den folgenden 2 Dezennien, also im Alter zwischen 30-50 Jahren. Nach der Statistik von Edgren verbält sich das weibliche Geschlecht vis-à-vis dem Flexurvolvulus in derselben Weise, nur daß hier noch im 6. Dezennium eine bedeutende Frequenz zu verzeichnen ist. Das Alter kann deshalb bei weiblichen Individuen kaum differentialdiagnostisch verwertet werden.

Daß gerade junge, in ihrem kräftigsten Alter stehende Personen so oft an Coecumvolvulus erkranken, beruht wohl darauf, daß die Patienten in diesem Alter mechanischen Momenten, wie heftigen Bewegungen, Traumata usw., wodurch eine Verlagerung des beweglichen Coecum zustande kommen kann, besonders ausgesetzt sind. In den beiden ersten hier mitgeteilten Fällen ist eine brüske Bewegung, wie sie beim kräftigen Hauen ausgeführt wird, ohne Zweifel das auslösende Moment gewesen, indem das Coecum dadurch in eine abnorme Lage geschleudert und gedreht wurde. Auch in dem Falle 3 muß wohl die vornübergebeugte Stellung des Patienten bei der Arbeit zur Verlagerung des Blinddarms beigetragen haben. Die nächste Ursache zu der bedeutenden und baldigst zu Gangrän führenden Drehung (um 450 ) im Falle 4, wo der Patient mitten in der Nacht durch die außerordentlich heftig einsetzenden Okklusionssymptome aus seinem Schlaf geweckt wurde, bleibt recht unerklärlich. In meiner früheren Zusammenstellung befinden sich 7 Fälle, wo ebenfalls die Patienten in der Nacht während des Schlafes von der Krankheit überfallen wurden. In dem Falle 5 ist gleichfalls die nächste Ursache zum Volvulus vollkommen dunkel. Die langjährige Verstopfung in diesem Falle, ebenso wie die aus unbekannter Ursache entstandenen Verwachsungen im vorigen Falle, hatten wahrscheinlich einen so labilen Zustand geschaffen, daß schon kleinere Schwankungen, z. B. im Fül- 
lungsgrad oder in der Lage der Därme ausreichten, einen fatalen Anfall auszulösen. Inwiefern Wilms und Payrs interessante Auslegungen zur Erklärung der Achsendrehungen des Dünndarms resp. der Stieltorsionen auch auf diese Fälle und auf den Coecumvolvulus überhaupt übertragbar sind, darauf will ich hier nicht näher eingehen.

Wenn ein junger Mann plötzlich mit Bauchschmerzen, Erbrechen und Verstopfung erkrankt, nachdem er schon früher mehrmals ähnliche schwerere oder leichtere Anfälle durehgemacht hat, wenn ein schräg oder quer verlaufender lokaler Meteorismus, gewöhnlich mit erloschener Peristaltik sichtbar ist, wenn der Bauch keine nennens. werte Druckempfindlichkeit aufweist, wenn per rectum eine so große Quantität Wasser eingegossen werden kann, daß ein tief belegenes Dickdarmhindernis ausgeschlossen werden $\operatorname{kann}^{1}$ ), wenn weiter der Verlauf verhältnismäßig chronisch ist und das Erbrechen, welches für eine Zeit aufhören kann, erst spät einen fäkulenten Charakter bekommt, da hat man, wie ich in meiner mehrmals zitierten Arbeit schon hervorgehoben habe, das typische Bild eines Volvulus des Coecum. Die verschiedenen Details dieses Schema können variieren und einige können sogar vollkommen fehlen. Doch sind in den allermeisten Fällen so viele von diesen Details zu finden, daß das Bild erkennbar wird und die Diagnose auf Volvulus coeci mit großer Sicherheit gestellt werden kann. Es scheint mir vollkommen berechtigt, den Coecumvolvulus als eine ebenso gut charakterisierte Krankheit wie z. B. den Flexurvolvulus, die Invagination und den Gallsteinsileus aus der Rumpelkammer des Ileus auszuscheiden und zu fordern, daß in der Zukunft diese spezielle Diagnose öfter gestellt werde. Bis jetzt scheint eine richtige Diagnose nur in sehr wenigen Fällen vor der Operation gestellt worden zu sein (Z. v. Manteuffel, Wanach, Faltin).

Von den hier mitgeteilten Fällen waren namentlich Nr. 1 und 3 besonders typisch. Auch die übrigen, mit Ausnabme von Nr. 4, boten ein für den Coecumvolvulus recht charakteristisehes Bild dar. Der Fall 4 ist namentlich in der Beziebung recht ungewöhnlich, daß die Symptome in solcher foudroyanter Weise auftreten. Eine heftige Strangulation kann, wie bekannt, mitunter ein Bild hervorrufen, das zum Verwechseln einer Perforationsperitonitis ähnelt. Beim

1) D. h. mit der Sicherheit dies uberhaupt geschehen kann. Ich teile vollkommen die Ansicht Philipowicz', daß einzig aus der Größe des möglichen Wassereinlaufes keine sicheren Schlüsse in bezug auf den Sitz des Verschlusses gezogen werden können. 
Coecumvolvulus' sind die Strangulationssymptome selten besonders heftig. In meiner früheren Zusammenstellung von 79 Fällen befinden sich nur 3 Fälle, die als heftige Strangulationen bezeichnet werden können. Das Coecum /war in diesen Fällen schon binnen wenigen Stunden gangränös geworden. Später hat Blecher einen äußerst rapide verlaufenden Fall publiziert, wo außer dem Coecum der ganze Dünndarm und das Colon ascendens bis zum Colon transversum in die Drehung eingezogen waren.

Unter den für Coecumvolvulus ; charakteristischen' Symptomen, möchte ich hier einige etwas näher besprechen, weil sie offenbar noch nicht genügend gewürdigt sind. Was erstens die 0 bstipation betrifft, ist es mir schon früher aufgefallen, daß in einer großen Anzahl (ca. 20) Krankengeschichten angegeben ist, daß nach dem Erkranken noch ein oder mehrere Stühle resp. Abgang von Flatus oft mit Nachlaß der Symptome entweder spontan oder nach Klystieren erfolgten. Auch in 3 von diesen 5 Fällen war die Obstipation nicht absolut. Beim Volvulus anderer Darmteile ist die absolute Obstipation bekanntlich die Regel. Man darf wohl deswegen annehmen, daß eine relative Obstipation ziemlich häufig nur beim Coecumvolvulus vorkommt und kann diesen Umstand vielleicht gelegentlich differentialdiagnostisch verwerten.

Eine einzige für alle Fälle gültige Erklärung dieser Erscheinung gibt es kaum. Mitunter mag es sich um Täuschungen gehandelt haben, indem durch das Klystier eingefuihrte Luft als Flatus inponierte. Manchmal kann der abführende Dickdarm noch beträchtliche Fäzesmassen enthalten und die Entleerung derselben eine Erleichterung verschaffen. Für manche Fälle ist man aber gezwungen, einen zeitweise nicht vollständigen Verschluß des Darmlumens anzunehmen. Dies entspricht übrigens der oft genug beobachteten geringen Drehung des Darmes um die longitudinale Körperachse, d. b. um die Darm- resp. Mesenterialachse, wodurch in erster Linie die Verlegung des Darmrohrs zustande kommt. Meiner Meinung nach bildet nämlich die Verlagerung, d.h. die Drehungen um die sagittale und transversale Achse, in vielen Fällen von Coecumrolvulus, wenigstens im Anfang, die Hauptkomponente des gewöhnlich ziemlich komplizierten Drehungsprozesses.

Der lokale Meteorismus zeigt mitunter, wie namentlich in meinen beiden letzten Fällen besonders prägnant zum Vorschein kam, ein Verhalten, welchem, wie es scheint, die Autoren bis jetzt keine besondere Aufmerksamkeit geschenkt haben. Es ist dies das Plätschergeräusch, welches man bei schüttelnder oder stoßender Palpation 
des lokalen Meteorismus hervorbringen kann. Ohne noch eine genügende Erfahrung zu besitzen, ob dies Symptom nicht auch beim Volvulus anderer Darmteile ebenso prägnant auftreten kann, erscheint es mir doch sehr wahrscheinlich, daß das sackartige, infolge der Drehung mit flüssigem Kote und Gasen gefüllte Coecum besonders geeignet sein muß, um dieses Phänomen hervorzubringen. Seitdem ich auf dies Symptom aufmerksam geworden bin, habe ich danach bei einigen anderen akuten Darmokklusionen, wie Invaginationen und Strangabklemmungen gefahndet, aber ohne es zu finden. Dagegen findet man dieses Plätschergeräusch, wie bekannt, fast regelmäßig bei chronischen Obturationen, wie z. B. bei Darmstenosen, wenn die zuführenden Därme infolge des angestauten Darminhalts aufgetrieben sind.

Jedenfalls erscheint es mir der Mühe wert, künftighin auch auf dies Symptom zu achten. Wenn es bald nach der Erkrankung auftritt, spricht es, wenn nicbt einzig für Coecumvolvulus, so doch für einen Volvulus überbaupt, d. h. für einen Zustand, wodurch ein begrenzter Darmabschnitt mit Gas und dünnflüssigem Inhalt gefüllt wird. Dies trifft ja ganz besonders bei Strangulationen ein, beim Coecumvolvulus infolge des sackartigen Darmes und infolge Schlußfähigkeit der Valvula Bauhini, wahrscheinlich auch, wenn es sich um reine Obturationen handelt. Da das Coecum bei tiefsitzendem, namentlich chronischem Dickdarmverschluß fast stets gebläht ist und große Massen flüssigen Kotes enthalten kann (Ansch ütz, Kreuter), ist es nicht $\mathrm{zu}$ bezweifeln, daß ein Plätschergeräusch auch unter diesen Umständen zu erzeugen sei. Es ist diese Möglichkeit ebenfalls in Erwägung zu ziehen.

Immerhin ist es mit diesen Einschränkungen nicht unwahrscheinlich, daß dies Symptom, auch wenn der lokale Meteorismus infolge versteckter Lage des gedrehten Darmes fehlen wurde, die Diagnose einer Achsendrehung, namentlich der des Blinddarms, einmal wird sichern können.

Beim Studium der Kasuistik ist es mir aufgefallen, daß die Patienten während der Schmerzanfälle oder auch dauernd bestimmte, oft höchst merkwürdige Lagen einnahmen, weil sie selbst bemerkt hatten, daß die Schmerzen in dieser Lage am geringsten waren.

Treves erzählt von einem Patienten, der schon mebrmals Okklusionsanfälle dadurch glücklich überwunden hatte, daß er, sobald er erkrankte, eine bestimmte Lage einnahm. Beim letzten Anfall half die erprobte Methode nicht mehr und der Patient mußte ins Jenseits. Treves bemerkt hierzu, daß er es für sehr wohl möglich bält, daß eine frische Achsendrehung durch eine Lageveränderung oder irgend- 
eine heftige Bewegung rückgängig gemacht werden kann. Hunter beobachtete bei einem Patienten mit angeblichem Flexurvolvulus, daß, wenn der Patient sich von rechts nach links drehte, nahmen die Schmerzen zu, wenn er sich in der umgekehrten Richtung drehte, nabmen die Schmerzen ab. Hunter drehte augenblicklich seinen Patienten dreimal in der wohltuenden Richtung mit dem Resultat, daß der Patient frisch und gesund von seinem Lager aufstand. Ebensogut gelang ihm diese Methode in drei anderen angeblichen Fällen von Flexurvolvulus.

In meinen beiden hier mitgeteilten Fällen 1 und 2, namentlich in dem ersten, war die besprochene Influ ierbarkeit der Bauch schmerzen durch Lageveränderungen sehr deutlich ausgeprägt. Trotz mehrmaligen Umdrehens des Patienten glückte es nicht, die Symptome zum Verschwinden zu bringen. Dr. Sahlberg hat, inspiriert durch meine Arbeit, bei seinem Patienten (5) ebenfalls ohne Erfolg diese Umdrehungsmethode versucht. Trotz dieser Mißerfolge kann ich nur raten, bei passenden Fällen diese Methode zu versuchen. Wenn man auch nur ein einziges Mal einen Erfolg zu verzeichnen bätte, wäre das Verfahren an die Seite der übrigen bei Darmokklusionen anzuwendenden unblutigen Maßnahmen, wie Klystiere, Insufflationen, Magenausspülungen usw., zu stellen. Jedenfalls kann man bei gehöriger Vorsicht kaum einen Schaden damit anstiften.

Größere Bedeutung scheint mir indessen dem Verfahren wahrscheinlich in diagnostischer Hinsicht zuzukommen. Die gesteigerten Schmerzen beim Lagewechsel können schwerlich anders als durch vermehrte Zerrung des Mesenteriums erklärt werden. Bei Achsendrehungen, wo in den meisten Fällen das Mesenterium schon durch die Drehung eine gewisse Zerrung erfahren hat, muß der gedrehte Darm, wenn ihm noch eine Beweglichkeit zukommt, bei jedem Lagewechsel bestrebt sein, den tiefsten Punkt im Bauche einzunehmen und folglich mitunter das gedrehte und gespannte Mesenterium noch mehr zerren, bei anderer Lage im Gegenteil dasselbe entspannen. Je größer der allgemeine Meteorismus ist, desto kleiner wird die Möglichkeit für jegliche Bewegungsexkursionen seitens des gedrehten Darmes. Folglich werden auch Lageveränderungen weniger Einfluß auf die Schmerzen ausüben können.

Wenn es sich um eine Darmokklusionsart handelt, bei der das Mesenterium gar nicht beteiligt ist, wie z. B. eine reine Obturation infolge von Gallenstein, Striktur, Tumor usw., oder um innere eingeklemmte Brüche, wo der inkarzerierte Darm fixiert bleibt, ist ein Finfluß auf die Bauchschmerzen durch Lageveränderung nicht zu 
erwarten. Bei Strangabklemmung kann wahrscheinlich sowohl das eine wie das andere eintreffen, je nach den gerade vorhandenen Verhältnissen. In zwei von mir vor kurzem beobachteten Fällen von Strangabklemmung hatten Lageveränderungen keinen Einfluß auf die Schmerzen. In zwei Fällen von Invagination war dasselbe der Fall.

Nach dem oben Angeführten halte ich es nicht für unwahr scheinlich, daß durch Lageveränderungen influierbaren Bauchschmerzen eine gewisse differentialdiagnostische Bedeutung beigelegt werden kann, namentlich im Beginn der Krankheit. Beim positiven Ausfall des Drehungsversuches kommen diagnostisch in erster Linie Volvulus in Betracht, in zweiter Linie möglicherweise Strangabklemmungen. Eine Unabhängigkeit der Bauchschmerzen von Lageveränderungen spricht, wenn der allgemeine Meteorismus keinen allzu hohen Grad erreicht hat, gegen die genannten und für andere Arten von Darmverschluß.

Trotz des selr schlechten Allgemeinzustandes im Falle 2 schritt ich zur Laparotomie und nicht zur primären Enterostomie mit Verzicht auf einen radikalen Eingriff. Es scheint mir nämlich das einzig Richtige zu sein, wenn man die Diagnose auf einen Volvulus gestellt hat, stets die Detorsion zu versuchen, auch bei sebr schwer angegriffenen Patienten. Da man in diesem Falle zielbewußt handeln kann, ist es möglich, den Eingriff bedeutend schonender zu machen, als wenn man auf eine ungewisse Diagnose hin die Laparotomie machen würde. Der schlechte Allgemeinzustand bezw. der Kollaps kann ja zum Teil auf der durch die Zerrung des Mesenteriums verursachten Reflexklemmung auf das Herz beruhen und das beste Mittel, um diesen Kollaps zu heben, ist selbstverständlich die Retorsion des Volvulus. Die radikale Operation kann und muß also, meiner Meinung nach, wenn es sich um einen Volvulus handelt, noch in viel schlechteren Fällen mit Aussicht auf Erfolg gemacht werden, als in Fällen, wo die Diagnose ganz unsicher ist.

Mit Rücksicht auf die Behandlung des Falles 1 möchte ich noch einige Bemerkungen anschlielsen. Vor der Operation wurde nach Muster vieler englischen und amerikanischen Chirurgen $0,015 \mathrm{~g}$ Strychnin nitr. subkutan verabreicht. Es soll in diesen Dosen besonders gtunstig auf den Shok einwirken. Obwohl diese Eigenschaft des Mittels nach neueren Untersuchungen (Crile) zweifelhaft sein mag, habe ich es doch mitunter angewandt, da ich den Eindruck bekommen habe, daß es anregend auf die Darmperistaltik einwirkt. $\mathrm{Zu}$ diesem Zwecke wurde es in Dosen von 0,003-0,005 g mehrmals täglich verabreicht. 
Die Bauchwunde wurde mit Ausnahme des unteren Wundwinke] geschlossen und nur ein ca. $4 \mathrm{~cm}$ breiter Silbergazestreifen gegen das kleine Becken eingeführt. Ich vermied es absichtlich, größere Tampons in die Bauchhöhle einzuführen, trotzdem das Coecum gangränös war und ziemlich viel trübe Aszitesflüssigkeit vorhanden war. Mein Vorgehen basierte sich auf die Resultate der bakteriologischen Untersuchungen von Bruchwässern und intraperitonealen Ergüssen bei Darmokklusionen. Bekanntlich steht trotz mancher vorhandener Widersprüche soviel fest, daß man selbst bei stark blutigem, getrübtem und übelriechendem Exsudat, auch wenn der inkarzerierte oder gedrehte Darm vollkommen gangränös aussieht, in einer Reihe von Fällen weder mikroskopisch noch kulturell Bakterien aufzuweisen vermag. Dies haben mir ebenfalls eigene, sowohl experimentelle wie klinische Untersuchungen mehrfach bestätigt.

Ich kann mich deswegen nicht zu der von manchen Autoren gehuldigten Ansicht anschließen, daß schon leichte Zirkulationsstörungen oder eine wenigstens nicht zu makroskopischer Gangrän führende Schädigung der Darmwand genügen, um dieselbe für Bakterien durchlässig zu machen.

Die Darmserosa ist sehr widerstandskräftig gegen eine Durchwanderung von Bakterien. Wenn die Mucosa und Muscularis nekrotisch sind, ibre Färbbarkeit verloren haben und ganz von Bakterien durchwuchert sind, bildet die Serosa oft noch einen schützenden Wall gegen die Bakterieninvasion ins freie Peritoneum. Wenn auch schließlich einige Bakterien durchdringen, wie im Falle 2, wo aus mehreren Kubikzentimetern Aszitesflüssigkeit nur einzelne Kolonien aufgingen, so ist dies nicht von einer so großen Bedeutung, daß eine ausgiebige Drainage der Bauchhöhle deswegen indiziert wäre. Das Peritoneum verträgt bekanntlich eine gehörige Menge von Bakterien, ehe es zu einer tödlichen Durchwanderungsperitonitis (Tavel) kommt. Payr glaubt übrigens, daß der bei vielen Formen von Darmverschluß, besonders bei Strangulation, als Staungstranssudat aufzufasssende intraperitoneale Erguß durch seine mutmaßliche bakterizide Kraft eine Art Schutzvorrichtung gegen die nach Schădigung der Darmwand auftretende Bakterieninvasion in die Bauchhöhle darstellt. Die bakterizide Eigenschaft des Bruchwassers ist wie bekannt, schon vor Jahren bewiesen (Schloffer). Wenn keine makroskopische Perforation sichtbar und das Exsudat nicht gerade jauchig ist, wie bei einer perforativen Appendicitis, glaube ich demnach, daß man auch trotz der Anwesenheit brandiger Därme die Aszitesflüssigkeit als in praktischem Sinne steril betrachten kann. 
Es ist nach dem Gesagten selbstverständlich, daß ich eine Drainage der Bauchhöhle für vollkommen unnütz erachte in allen den Fällen von Darmokklusion, wo der Darm reponiert werden kann, aber eine, wenn auch noch so reichliche und trübe und sogar fade riechende. Aszitesflüssigkeit bei Injektion der Därme vorhanden ist. Nur in einzelnen Fällen, wo eine Resektion des Dickdarms notwendig wurde, und das Exsudat purulent oder jauchig ist, mag tamponiert werden.

Es gibt noch einen anderen Grund, um eine Drainage der Bauchhöhle mittelst großer Tampons nach Möglichkeit nach Operationen wegen Darmokkulusion zu vermeiden. Die Anwesenheit großer Tampons in der Bauchhöhle ist nämlich keineswegs indifferent und kann sicherlich als Fremdkörper, die nach jeder Operation wegen Darmokkulusion, noch mehr als die nach einer gewöhnlichen Laparotomie, stets zu befürchtende Darmparese nur steigern. Im Handbuch der praktischen Chir. Bd. III, S. 231 betonen ebenfalls Mikulicz und Kausch unter den Nachteilen der Tamponade der Bauchböhle, die Beeinträchtigung der Darmperistaltik infolge von den um den Tampon entstehenden Verklebungen. Olsh a u sen ist wie bekannt kein Freund der Tamponade.

Die Resultate einiger mittelst Tamponade behandelten Fälle von Darmokklusion, die ich Gelegenheit gehabt habe zu beobachten, sind auch nicht ermutigend gewesen. Der in meinem Falle 2 auf einige Tage eingeführte Gazestreifen war zu unbedeutend, um weder in der einen oder anderen Richtung wirken zu können und hătte am besten ganz vermieden werden sollen.

Die Entleerung des Darmes ist nach Beseitigung des Volvulus die nächste und wichtigste Aufgabe, um ein glückliches Resultat zu erreichen. Die Bedeutung dieses Teiles der Behandlung der Darmokklusionen ist in den letzten Jahren von Kocher, Helferich, Heidenhain, Kukula, Albeck, Hepner u. a. genügend hervorgehoben worden.

In meinen beiden Fällen ( 1 und 2) wurden Laxantia während 4 Tage nach der Operation verabreicht. Außerdem wurde im Falle 1 tägliche Klystiere und Darmausspülungen appliziert. Im Falle 2 wurde die Entleerung sehr durch den Anus praeternaturalis erleichtert. Dies war auch ein Grund, warum die Darmnaht nicht ausgeführt wurde. Ein zweiter Grund war natürlich die dadurch erheblich verkürzte Operationsdauer. Sahlberg (Fall 5) punktierte bei der Operation den Darm und gab einmal $15 \mathrm{~g}$ englisehes Salz. Nach den günstigen Erfahrungen, die ich mit der Verabreichung von Laxantia nach Operationen wegen Darmokkulusion gemacht habe, kann 
ich dies Verfahren nur aufs beste empfehlen. In der sämtlichen mir bekannten Kasuistik über Volvulus coeci befinden sich 11 Fälle, wo Laxantia nach der Operation verabreicht wurden. Nur ein einziger Patient starb. Es kann dieses gute Resultat nicht nur auf Zufall beruhen, denn es befinden sich unter diesen Fällen sowohl leichte, wie versäumte und schwere.

In der Nachbehandlung des Falles 2 gibt es einen Punkt, dem ich einen nicht unwesentlichen Anteil an dem glïcklichen Ausgange zuschreiben möchte. Es ist dies die genügende Wasserzufuhr unter Vermeidung des natürlichen Weges per os. Die Patienten vertragen bekanntlich in den ersten Tagen, ehe der Darm sich gründlich entleert und erholt hat, nicht gut weder Nahrung noch Flüssigkeit, wenn dieselben per os eingeflölt werden.

Um nicht eine Wasserverarmung herbeizuführen, teils auch um den Patienten direkt zu stimmulieren oder, nach der Ansicht namentlich französischer Autoren, um seinen mit Toxinen überladenen Körper auszuwaschen, muß man aber unbedingt dem Körper eine genügende Menge Flüssigkeit zuführen ${ }^{1)}$ Dies kann durch intravenöse oder subkutane Kochsalzinfusionen oder in Form von kleineren $(200-500 \mathrm{ccm}$ großen) warmen Klystieren oder großen Rektalausspülungen geschehen. Diese letzteren werden soappliziert, daß man von einer ca. $40^{\circ} \mathrm{C}$. warmen Kochsalzlösung durch einen gewöhnlichen, mit einem Trichter verbundenen Magenschlauch ca. 1 Liter oder mehr auf einmal langsam einfließen läßt. Ohne den Schlauch herauszuziehen, läßt man die Flüssigkeit wieder herausfließen. Dabei entleeren sich oft Flatus und etwas Fäzes. Diese Prozedur, wird mehrmals wiederholt, bis 5-10 Liter verwendet worden sind. Zuletzt wird gewöhnlich etwas Flüssigkeit im Rektum gelassen. Während der Spülung wird schon ziemlich viel Flïssigkeit resorbiert. Diese Prozedur hat leider den Nachteil, daß die meisten Patienten sich dadurch ziemlich angegriffen und belästigt fühlen. Auch die kleinen Wassereinläufe von $200-500 \mathrm{ccm}$ werden mitunter auf die Dauer nicht gut vertragen. Noch weniger angenehm für die Patienten sind die subkutanen und intravenösen Infusionen. Diese Unannehmlichkeiten sind doch von verschwindend untergeordneter Bedeutung im Vergleich zu der außerordentlich günstigen Wirkung, die man namentlich mit intravenösen Infusionen bei sehr elenden Patienten erzielen kann. Immerhin kann es in manchen Fällen, wo man lange Zeit hindurch gezwungen ist, dem

1) Eine eingehende Darstellung dieser Gesichtspunkte findet sich in der interessanten Arbeit von Friedrich (Zur bakteriellen Ätiologie und zur Behandlung der diffusen Peritonitis. v. Langenbecks Archiv. 68. Bd. Heft 2). 
Patienten Kochsalzlösung beizubringen, eintreffen, daß einem schließlich keine weiteren Venen mehr zur Verfügung stehen. Dann kann es von einigem Nutzen sein, noch andere Applikationsmethoden zur Hand zu haben. In meinem Falle $2 \mathrm{kam}$ ich auf die Idee, den eingenähten Stumpf des Colon ascendens hierzu zu benutzen. Mittelst eines im Darmlumen tief eingeschobenen weichen Katheters konnte man den Patienten ohne Unannehmlichkeiten ca. 1/2 Liter Flüssigkeit auf einmal eingießen. Der Patient fühlte sich dadurch stets belebt und das Durstgefühl wurde gehoben. Bei einem anderen Patienten, dem wegen einer Invaginatio ileo-colica das Coecum und ein Stïck Ileum reseziert worden war, habe ich später dasselbe Verfahren angewandt. Es wurde diesem Patienten während 3 Wochen fast täglich 1 bis mebrere Liter Flüssigkeit in Gaben von ca. 1 Liter auf diesem Wege beigebracht. Leider starb Patient später an Peritonitis, ausgehend von einer Perforation am zuführenden Darm, ohne daß die Applikationsweise irgendwie dafür beschuldigt werden konnte. Das erwähnte Verfahren scheint mir jedenfalls der Nachprüfung wert zu sein. Die Fälle, welche sich dafür besonders eignen, sind solche, bei denen nach Coecumresektion ein Anus praeternaturalis besteht. Dies kommt z. B. bei den nicht allzu seltenen Fällen vor, wo man nach der Empfehlung von Mikulicz eine zweizeitige Coecumresektion gemacht hat.

Nach meiner früheren Zusammenstellung waren von 75 Fällen $28(=34,44$ Proz.) geheilt und $51(=64,55$ Proz.) gestorben. In 20 Fällen wurde die Detorsion des Volvulus nicht gemacht. Alle starben. Wenn diese Fälle ausgeschaltet werden, erhält man unter 59 mit Detorsion behandelten Fällen $28(=47,54$ Proz.) Heilungen, d. h. fast jeder zweite Patient genas.

Im ganzen ist die Resektion des brandigen Coecum 9 mal ausgefübrt worden. Nur drei Patienten (Z. v. Manteuffel, Fick, Faltin) kamen mit dem Leben davon.

Obwohl die Resultate bis jetzt nicht besonders glänzend gewesen sind, glaube ich doch, daß der Coecumvolvulus in der Zukunft zu den Okklusionsformen gerechnet werden wird, für welche man eine relativ günstige Prognose stellen kann. Diese, meine, schon früher anderswo ausgesprochene Ansicht, gründet sich nicht nur auf die Kenntnis des im allgemeinen gutartigen Verlaufes der Krankheit, sondern besonders auf meine Überzeugung, daß3 der Coecumvolvulus in den allermeisten Fällen ein genügend charakteristisches klinisches Bild aufweist, um diagnostiziert werden zu können und dadurch ein zielbewußtes, erfolgreiches Handeln zu ermöglichen. Immerhin werden 
stets einige Fälle vorkommen, die infolge ungewöhnlicher Darm- und Drehungsverhältnisse oder infolge hyperakuten Verlaufes nicht erkannt werden können oder von vornherein als verloren angesehen werden müssen.

\section{Literaturverzeichnis.}

Anschütz, Über den Verlauf des lleus bei Darmkarzinom und den lokalen Meteorismus des Coecum bei tiefsitzendem Dickdarmverschluß. v. Langenbecks Archiv. 68. Bd. Heft 1. 1902.

Hepner, Zur Diagnostik und Therapie des inneren Darmverschlusses. Beiträge z. klin. Chirurgie. 36. Bd. 1902.

Kreuter, Dehnungsgangrän des Coecum bei Achsendrehung der Flexura sigmoidea und bei Abknickung des Blinddarms. v. Langenbecks Archiv. 70. Bd. Heft 2. 1903.

K u hn, Über Volvulus der Flexura sigmoidea. Beiträge z. klin. Chir. 36. Bd. 1902. Edgren, Volvulus flexurae sigmoideae. Dissertation. Helsingfors 1901.

Faltin, Beiträge zur Kenntnis des Volvulus coeci. Nordiskt med. Arkiv. Abteilung Chirurgie. 1902. Heft 4. 1903. Heft 1 u. 2. Anh.

Payr; Über die Ursachen der Stieldrehung intraperitoneal gelegener Organe. v. Langenbecks Archiv. 68. Bd. 1902.

Derselbe, Über ausgedehnte Darmresektionen. Bemerkungen über chemische Peritonitis und das Stauungstranssudat bei Strangulationsileus. v. Langenbecks Archiv. 67. Bd. 1902.

Wilms, Wie entstehen Achsendrehungen (Volvulus) des Dünndarms. v. Langenbecks Archiv. 69. Bd. 1903.

Derselbe, Mechanismus der Knotenbildung des menschlichen Darmes. v. Langenbecks Archiv. 69. Bd. 1903.

Derselbe, Mechanismus der Strangulation des Darmes. Deutsche med. Wochenschr. 29. Jan. 1903.

Hier möchte ich noch einige Arbeiten anführen, in denen Fälle von Coecunvolvulus beschrieben sind. Diese Fälle sind mit einer Ausnahme nach Abschlul meiner früheren Arbeit publiziert worden.

Blecher, Über Ileus bedingt durch seltenere Formen von Volvulus. Deutsche Zeitschr. f. Chirurgie. 64. Bd. 1902.

Burgers, The Lancet. 1902. Dec. 20.

Harsl of , 4 Tilfaelde af Ileus. Hospitaltidende. No. 28. 1903.

Johan Nic olaysen, Studier over lleus. Norsk magazin for Laegevidenskaben. Juni 1895. Anhang.

Karström, Sechs Fälle von Ileus. IIygiea. 1902. II. 4, S. 477.

Kreuter 1. c.

Fröhlich, Deux observations d'occlusion par vice de position de l'intestin. Bull. et mém. de la soc. de Chir. de Paris. Tome 27. p. s42.

P'escatore, Ein Fall von Volvulus bei einem 19tägigen Säugling. Deutsche Zeitschr. f. Chirurgie. 68. Bd. 1903.

Philipowicz, Mitteilungen über inneren Darmversehluß. v. Langenbecks Archiv, 70. Bd. 1903.i

Wandel, Über Volvulus des Coecum und Colon ascendens. Mitteil. a. d. Grenzgeb. d. Med. u. Chir. 11. Bd. 1903. Heft 1. 